\title{
Let others manufacture! Towards a new manufacturing framework
}

José Ramón Vilana Arto

Universidad Politécnica de Madrid

(Spain)

jrvajrva@gmail.com
Carlos Rodriguez Monroy

Universidad Politécnica de Madrid

(Spain)

crmonroy@ingor.etsii.upm.es

Received October, 2009

Accepted November, 2009

\section{Abstract:}

Global manufacturing virtual networks (GMVNs) constitute a new type of vertical and horizontal relations between independent companies or even competitors where it is not needed to maintain internal manufacturing resources but to manage and share the network resources. The fluid relations that exist within the GMVNs allow them a very permeable organization easy to connect and disconnect from one to each other as well as to choose a set of partners with specific attributes. The result is a highly flexible system characterized by low barriers to entry and exit, geographic flexibility, low costs, rapid technological diffusion, high diversification through contract manufacturers and exceptional economies of scale and specialization. This paper analyses the causes behind the formation of such networks, their strategy, structure, dynamics and culture, taking into account areas such as strategic alliances between competitors, the permeable and diffuse nature of the network actors, the inherent paradox of collaborating with competitors, cross-cultural issues or information and communication technologies at the network level. This work will clarify and put these organizations in perspective and will analyze their evolution over the next few years.

Keywords: Global Manufacturing Virtual Networks, strategic alliances, contract manufacturing, cross-culture.

JEL Code: 014 


\section{Introduction and theoretical framework}

Today, the concept of plant or production centre is becoming increasingly more ambiguous. In many industries, there is growing collaboration between production centres and manufacturing networks that seek to respond to market demands more efficiently and obtain competitive advantages in a globalized environment. In some industries, such as the aeronautical industry, the electronics industry or the automotive industry, there is mention of Global Manufacturing Virtual Networks (GMVNs) based on a new manufacturing architecture model with a high development potential to satisfy an increasingly demanding and fragmented market (Shi et al., 2001).

The global environment, in which companies presently operate, with ever more globalized markets, consolidations of companies or strategic alliances, is forcing firms into finding new ways of collaboration that would improve the integration and synchronization of the different functions and stages of the value chain of their products. Within these networks, the suppliers maintain close relations with a very varied number of clients permitting them to achieve better economies of scale and also minimizing the risk of losing a specific collaboration with one of them (Fine, 1998). On the other hand, the OEMs maintain relations with an interchangeable group of suppliers according to different technical and geographical particularities. GMVNs minimize the almost exclusive interdependence between the OEM and the suppliers which existed in the first phases of disintegration of the value chains (Elmuti et al., 2001). The result is a network with a very permeable and flexible structure, with very fluent relations and very low entrance and exit barriers, permitting a very rapid diffusion of technology and very high economies of scale. While the value chain of a company defines the vertical sequence of sequential activities permitting a particular product or service to be produced, a GMVN consists of several value chains (one for each actor participating in the network) including relations of the vertical and horizontal type and which are continually and dynamically being reconfigured (Sturgeon, 2000). In this context, a value chain could be considered as a sub-unit of a GMVN, more static and determined than the latter, though much easier to represent and define.

However, although the potential of these organizations is evident they also reveal a number of problems that must be solved to ensure their own survival. A key 


\section{Intangible Capital}

feature of GMVNs are horizontal relationships among its members, in many cases direct competitors, that decide to collaborate on a specific project even though they compete aggressively in other projects or other phases of their value chains.

There are many examples of such collaboration and competition relationship. NEC and Siemens collaborate in the field of $R \& D$ and compete aggressively to commercialize the products developed under this collaboration. Philips and Sony collaborate on the development and manufacture of new DVDs, while compete very aggressively in other products and markets (Luo, 2007). The manufacture of some aeronautical engines involves the participation of the great majority of manufacturers (competitors) in the market. For example, the GP 7200 engine that powers the new Airbus 380 is manufactured by an alliance between General Electric and Pratt \& Whitney with collaborations from MTU Aero Engines, Snecma, and Tech Space Aero. This paradox is permitted by the OEMs (Original Equipment Manufacturers) because the benefits obtained through this collaboration are much greater than the inherent risks of collaborating with competitors.

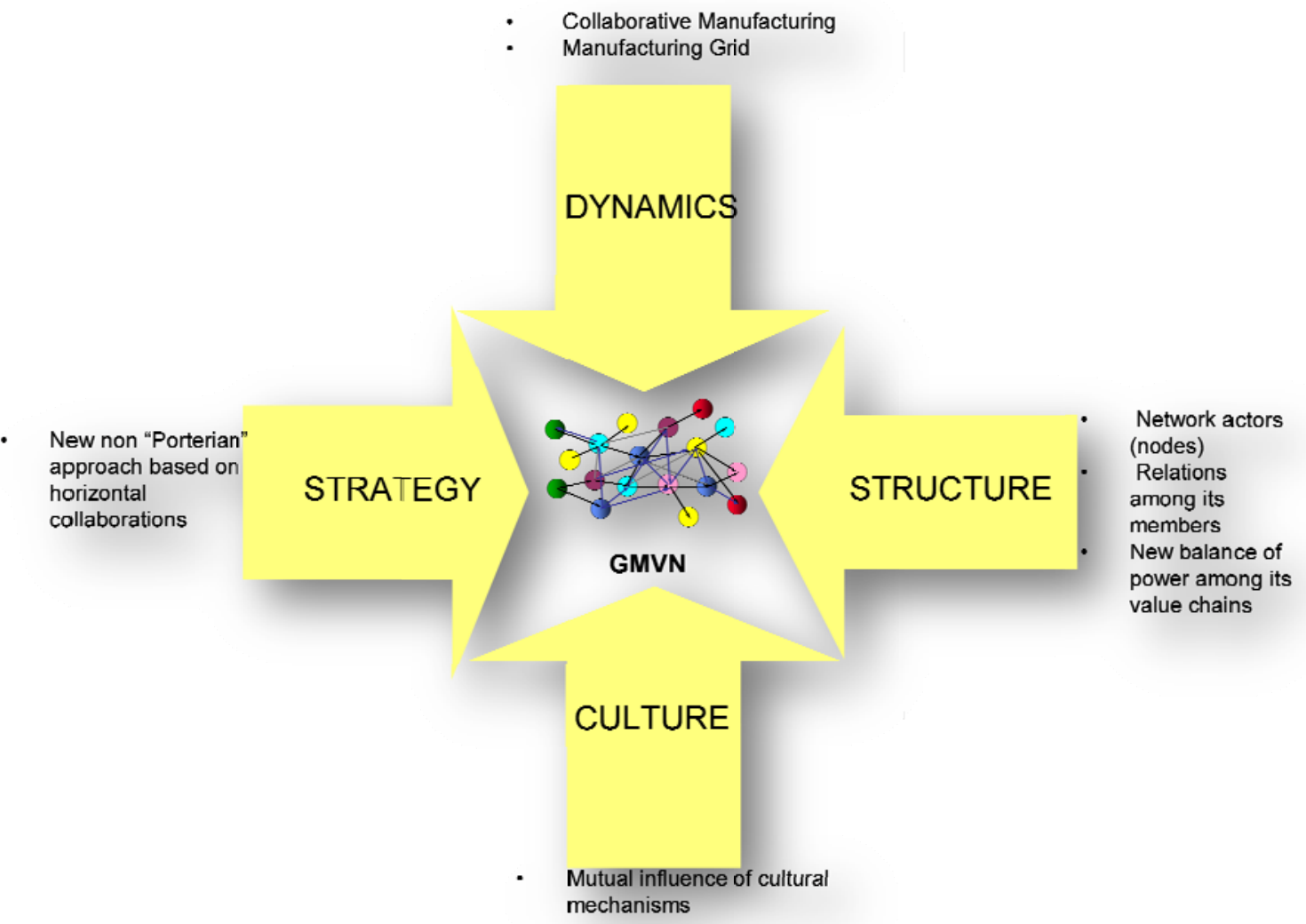

Figure 1. Basics Aspects of GMVNs 
The network structure feature includes the performance of its main actors (the nodes of the network), as well as the type of relations and collaborations that are established among its members. The nature of its actors and their relations is not anything static and rigid but a system undergoing a continual process of change with, in some cases, diffuse and variable structure.

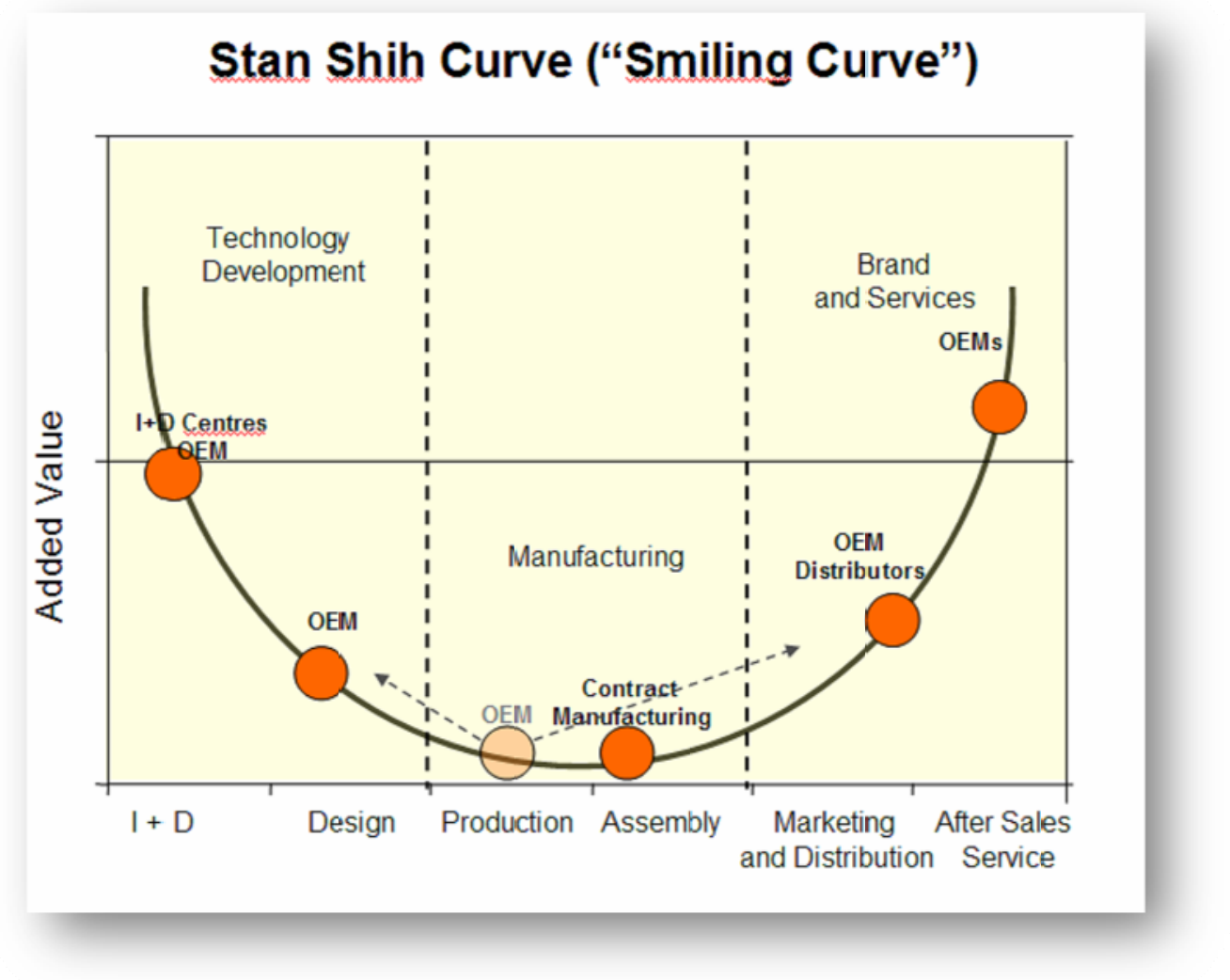

Figure 2: Location of GMVN actors in the Stan Shih curve

A clear manner to understand the origin of GMVNs, the different roles of their actors and their progress is through their representation in the Stan Shih curve shown in Figure 2. Stan Shih (founder of the computer manufacturer Acer) linked the different sequential stages of the value chain of a product or service with the added value they bring to the company. According to this chart, the extremes of the value chain are those that bring more value, $R \& D$, marketing and after sales services processes, while the central stages related to manufacturing and assembly processes provide less value Although the original purpose of Stan Shih was to apply this curve to the computer industry, its principles can be applied in most of GMVNs cases. OEMs within GMVNs move away from manufacturing and assembly 
processes, which are the stages that provide them less value and invest in higher value stages such as $R \& D$ or marketing, to constitute its core competencies. At the same time, manufacturing and assembly functions are given to new players in the network: the contract manufacturers. This approach can question the interest of contract manufacturers in these tasks when the OEM itself, which is the best who knows the manufacturing processes, decides to leave them. The answer would be that Stan Shih curve presented here is referred to the point of view of the OEM. The contract manufacturer achieves much higher economies of scale than the OEM because he produces similar products for other OEMs in some cases competitors. Therefore, from the point of view of the contract manufacturer, manufacturing and assembly stages will provide him a much higher value than the one perceived by a single OEM. The problem arises when the contract manufacturer is moving forward in its learning curve and accessing to new know-how technology that can be used in developing its own products. Therefore, a natural tendency of the contract manufacturer will be to manufacture its own products becoming an OEM competitor of its former customers. The former contract manufacturer Lenovo is a good example of this conversion into OEM by using its own distribution channels and becoming one of the leaders within the computers sector. Recently, the AustrianCanadian contract manufacturer Magna that manufactures and assembles cars for Mercedes, BMW and Saab announced its interest to buy Opel which will imply a significant technology transfer and the adaptation of Magna as a new OEM that will compete with its former customers. Another possibility is when a contract manufacturer becomes an OEM but uses the brand and distribution channel of wellimplanted retailers in the market. Thus, large retail chains like Wal Mart or Carrefour may order products like cars to contract manufacturers under their own distribution brand with the same quality as the major brands but at significantly lower prices. In the coming years, this process will occur in products that traditionally were only OEM branded (Arruñada, 2006).

Figure 3 shows the different types of collaboration that can occur within the GMVNs. The first one is the simplest case where two or more actors in the network establish a partnership of collaborative manufacturing. Rolls Royce manufactures the Trent 900 aircraft engine, designed to power the new Airbus 380, with the collaboration of several companies because of the financial risk involved and the high technological complexity of the project. Some of these partners are Volvo 
Aero that makes the intermediate compressor casing, ITP responsible for the lowpressure turbine or Honeywell in charge of the pneumatic systems.

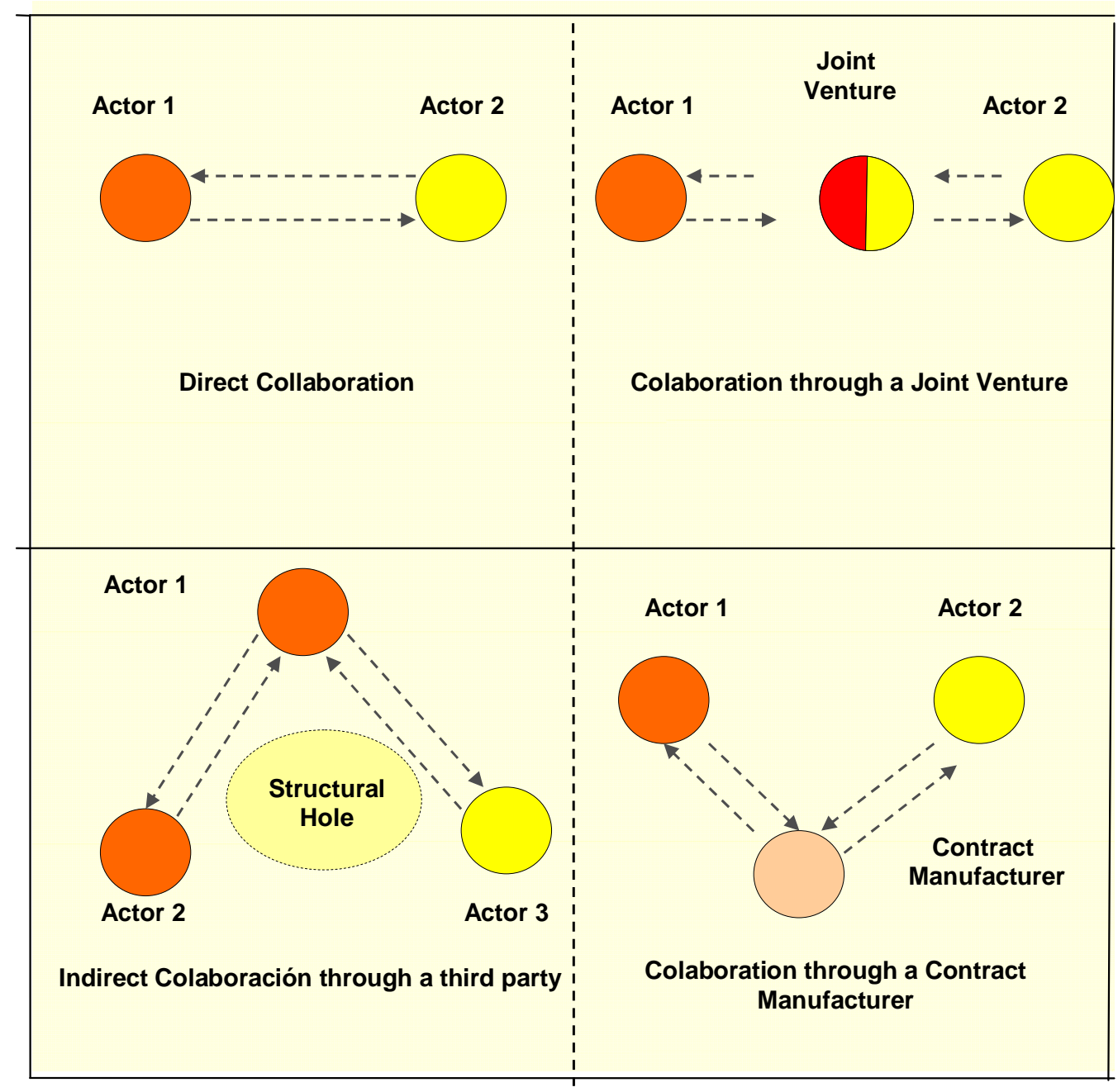

Figure 3. Types of Collaboration among GMVN Actors

In the second case, cooperation is made through the establishment of a joint venture. The partnership developed between General Electric and SNECMA to manufacture the CFM 56 engine was developed through a new 50-50 joint venture named CFM International. This kind of collaboration is very usual between companies competing in the same sector since minimizes opportunistic behavior and prevents undesirable technology transfer (Gulati, 1995). A third type of collaboration is through the so-called structural holes (Burt, 1992). This happens when two or more firms have relation with the same actor without existing relationships between them. This mechanism promotes diversity and new 
information flows to the focal actor since avoid duplication and overlapping relationships. It is very useful in consulting firms or new products development networks. An alternative to this case is an indirect collaboration through a contract manufacturer. This relationship is allowed by the two actors who collaborate with the contract manufacturer because of the benefits they get from this collaboration are greater than the inherent risk of collaborating with competitors. Ericsson and Motorola collaborated indirectly through the contract manufacturer Flextronics to manufacture some electronic components because of the enormous economies of scale they achieved.

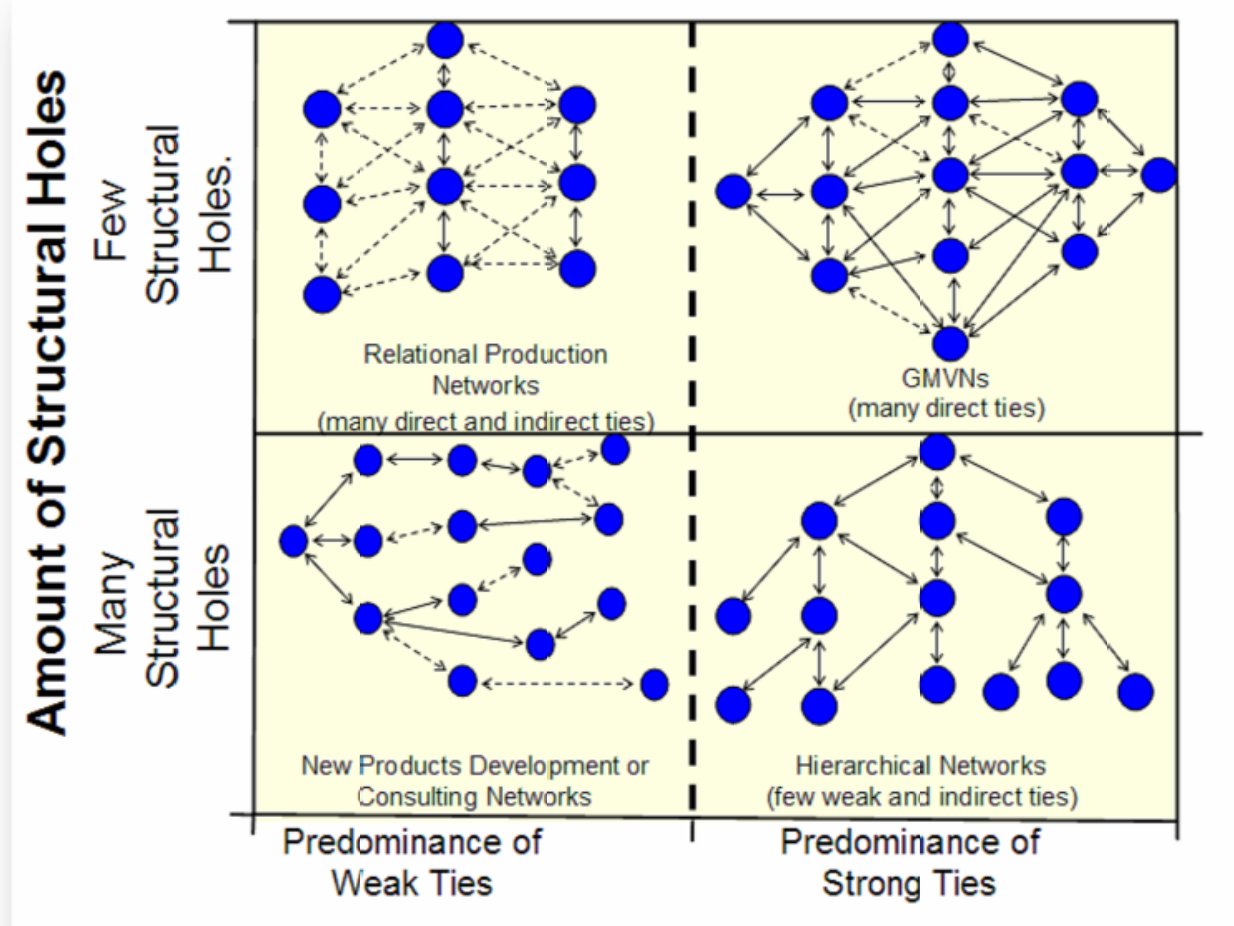

\section{Strong vs. Weak Ties}

Figure 4. Types of Networks

There are three kind of network attributes that strongly influence the structure of GMVNs. These attributes are: 1) strong ties versus weak ties (Granovetter, 1985), referred to the intensity of collaboration between two companies, 2) direct versus indirect ties (Ahuja, 2000), two companies can collaborate with each other directly or through a third actor and 3) structural holes (Burt, 1992). Depending on the 
combination of these attributes, different network typologies can be obtained. Figure 4 summarizes the most general type of networks. Networks composed of relationships with partners with few ties to others are characterized to have many structural holes and few direct and indirect ties. This typology facilitates control over exchange partners like the case of hierarchical networks of suppliers widely used in the 80's by Japanese companies in the automotive sector. Networks with many interconnected relationships between all the actors are characterized by few structural holes and high intensity of strong ties, which promotes trust and cooperation among their actors to avoid positions of power (Ahuja, 2000). This type of organization is usual on high-tech networks with much horizontal collaboration. Networks with few overlaps and redundancies are characterized by having many structural holes and predominance of weak ties that provide benefits access to new information. Such organizations are ideal for brokerage of information or technology like the consulting networks. Finally, networks with many interactions between all their members through weak ties are what Sturgeon (2002) called relational production networks. They are characterized by intense social ties that underlie the economic network. Granovetter (1985) defined this phenomenon as social embeddedness. GMVNs, due to the strong component of horizontal relationships between competitors, should have a closed typology with numerous and intense relations between their actors, with a greater percentage of direct ties that encourage resource sharing and access to tacit knowledge to promote the development of trust.

Proposition 1: The typology of GMVNs is mainly characterized by few structural holes with predominantly strong and direct ties.

Many authors (e.g., Granovetter, 1985; Hofstede et al., 1990) have analyzed the presence, under a systemic perspective, of some homogeneous cultural values and practices where network actors can be identified to strengthen the group membership or establish a social network that underlies the own GMVN and facilitates interactions among its members. The feasibility of this approach would facilitate the formation of new GMVNs by establishing, ex ante, a cultural prescriptive model at the network level. The chart in figure 5 shows this approach through four cultural mechanisms: the similarity of cultural practices among its actors, social embeddedness, transfer of tacit knowledge and the importance of trust in the network. 
There is a continuous interaction of these four cultural mechanisms during the whole duration of the relationship by creating a continuous and mutual reinforcing process. Although they come from the discipline of organizational culture, they have a different meaning within GMVNs. They are divided according to their influence before or after the formalization of the relationship in mechanisms ex ante and ex post. Ex ante mechanisms condition the formation of the GMVN by determining the energy that will be necessary for establishing that relationship. These mechanisms are the similarities of cultural practices and social embeddedness. Ex post mechanisms facilitate and enhance the interaction of actors in the network once the relationship is formed and they are tacit knowledge transfer and trust.

There is a continuous interaction of these four cultural mechanisms during the whole duration of the relationship by creating a continuous and mutual reinforcing process. Although they come from the discipline of organizational culture, they have a different meaning within GMVNs. They are divided according to their influence before or after the formalization of the relationship in mechanisms ex ante and ex post. Ex ante mechanisms condition the formation of the GMVN by determining the energy that will be necessary for establishing that relationship. These mechanisms are the similarities of cultural practices and social embeddedness. Ex post mechanisms facilitate and enhance the interaction of actors in the network once the relationship is formed and they are tacit knowledge transfer and trust.

It seems that there is a consensus among most of scholars (e.g., Hofsteted et al., 1990; Gulati, 1995; Gupta \& Govindarajan, 2000) about the importance of cultural similarities in inter-firm collaborations to conclude that participants with similar cultural practices reach high assessments of satisfaction, learning and collaboration efficiency. On the other side, companies that have strong differences in their organizational culture must expend enormous energy and time to establish management practices and organizational routines to facilitate their interaction (Pothukuchi et al., 2002). The problem arises when, once a GMVN collaboration is formed, strong inconsistencies are detected in one or more cultural practices. In fact, it is exceptional to find a potential collaborator with a strong compatibility and similarity of all cultural practices since they do not constitute a cause of such collaboration but are a necessary condition for its success. The collaboration 


\section{Intangible Capital}

between GE and SNECMA for the aircraft engine manufacturing CFM 56, partially solved this dilemma, by constituting some new ad hoc departments and internal processes, identical in both companies, exclusively related to the new program for developing the CFM engine. This measure allowed the formation of subcultures in these departments with high degree of cultural practices similarity between the two companies. Later, it was formed a new $50-50 \%$ joint venture that facilitated the cultural compatibility in their practices.

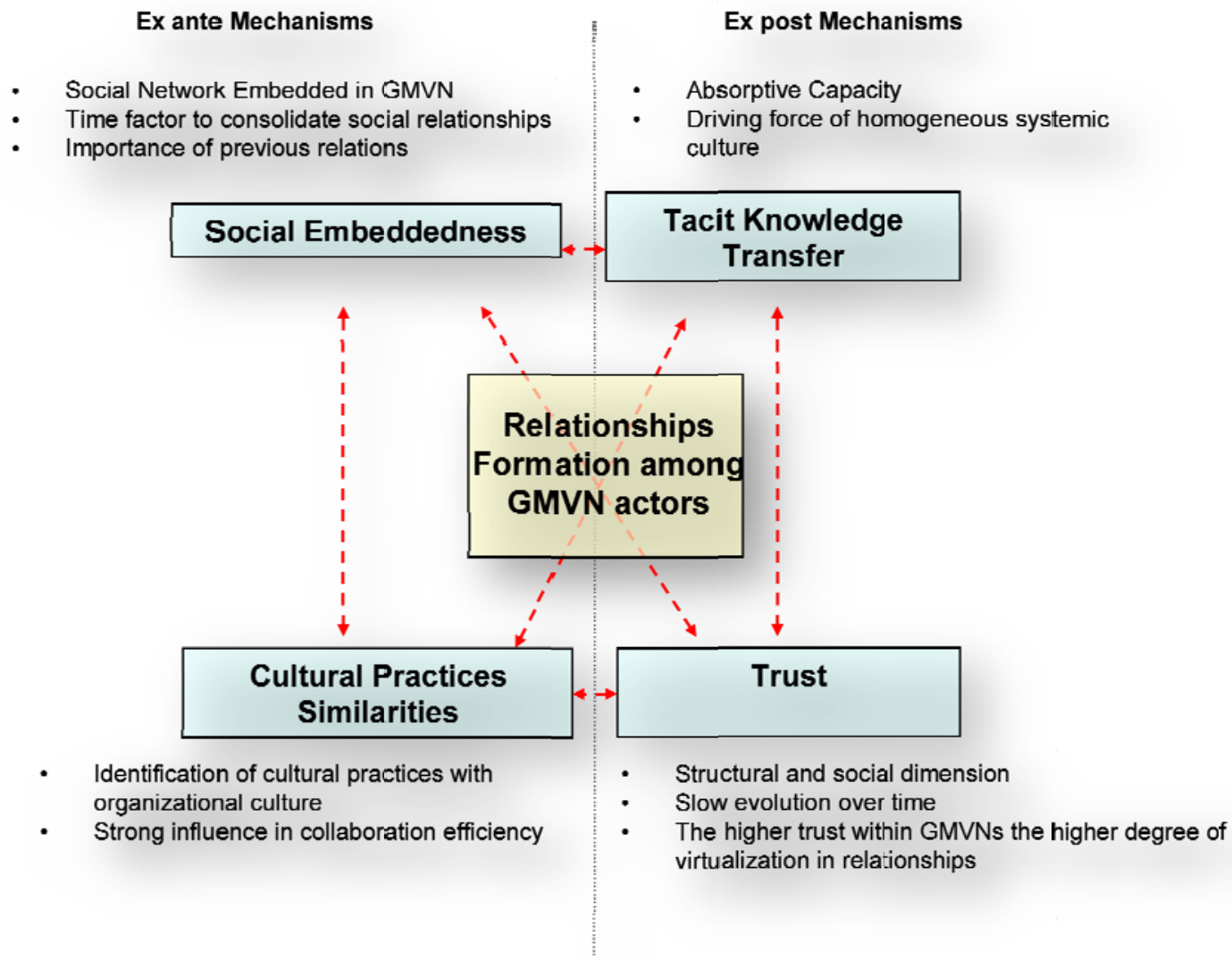

Figure 5: Cultural Mechanisms in GMVNs

Social embeddedness is another ex ante mechanism that favors the formation of GMVNs. These organizations are embedded in a social context of previous relationships that create a social network in which the companies are integrated and represent a source of invaluable information about the reliability and capacity of current and potential participants in the GMVN (Gulati, 1995). Although formation of this social network takes time, it is a cumulative process that builds on past experience. Therefore, social embeddedness gives information to the stakeholders of GMVNs on skills, needs and competencies of potential new 


\section{Intangible Capital}

(c) (c) Intangible Capital, 2009 - 5(4):347-369 - ISSN: 1697-9818

collaborations in the network and increases reliability against potential opportunistic behavior. These social networks need time to settle and once established they will act as a catalyst for business relations in the network (Gulati $\&$ Gargulio, 1999). To know the map of social relationships on the network is a key factor to succeed in GMVNs. It is very complex to start a close collaboration from the beginning if there is not a previous experience of less demanding relationships that allow constructing a network of embedded social relations in the GMVN.

Tacit knowledge transfer is an ex post cultural mechanism that satisfies many of the demands of GMVNs. One of the reasons to exist these networks is the ability to transfer and make use of knowledge more efficiently in an environment of alliances rather than through external market mechanisms. Anyhow, this argument does not mean that tacit knowledge is easily transferred through the network since this fact will be determined by the collaborative and competitive position of the network actors. This type of knowledge represents one of the most efficient competitive advantages sustainable over time (Gupta \& Govindarajan 2000). It is also one of the main factors that helps to differentiate companies from each other. There are many possibilities that a strong tacit knowledge transfer between two network actors produce a convergence in their cultural practices and consequently this fact will help to homogenize culturally the network. Thus, it can be affirmed that tacit knowledge transfer in a GMVN can help to build a systemic culture. When a network actor shares with other participants, a kind of knowledge that is one of its most important assets, what determine its own identity and distinguishes him from other network actors, when this asset is diluted and expanded through the GMVN, a process of cultural homogenization start to occur in the network.

Trust is a cultural ex post mechanism in GMVNs which evolves slowly over time and if the appropriate measures are taken it can notably reduce transaction costs (Gulati \& Gargulio, 1999), make more fluid business collaboration and facilitate tacit knowledge transfer in the GMVNs. The graph in Figure 6 shows the various stages of trust in relations between companies within a GMVN and its relationship to the degree of "virtualization".

Cultural mechanisms significantly influence the formation and survival of GMVNs by exercising a number of cultural benefits. These benefits are 1) easy integration of new members or collaborators in a GMVN based primarily on social embeddedness, 
like references or past experiences of one or more actors in the network, 2) increase of a homogeneous culture increasingly strengthened due mainly to the social network of relationships that were established, similarity of cultural practices and tacit knowledge transfer and 3 ) reduced transaction costs caused mainly by increased trust between network actors.

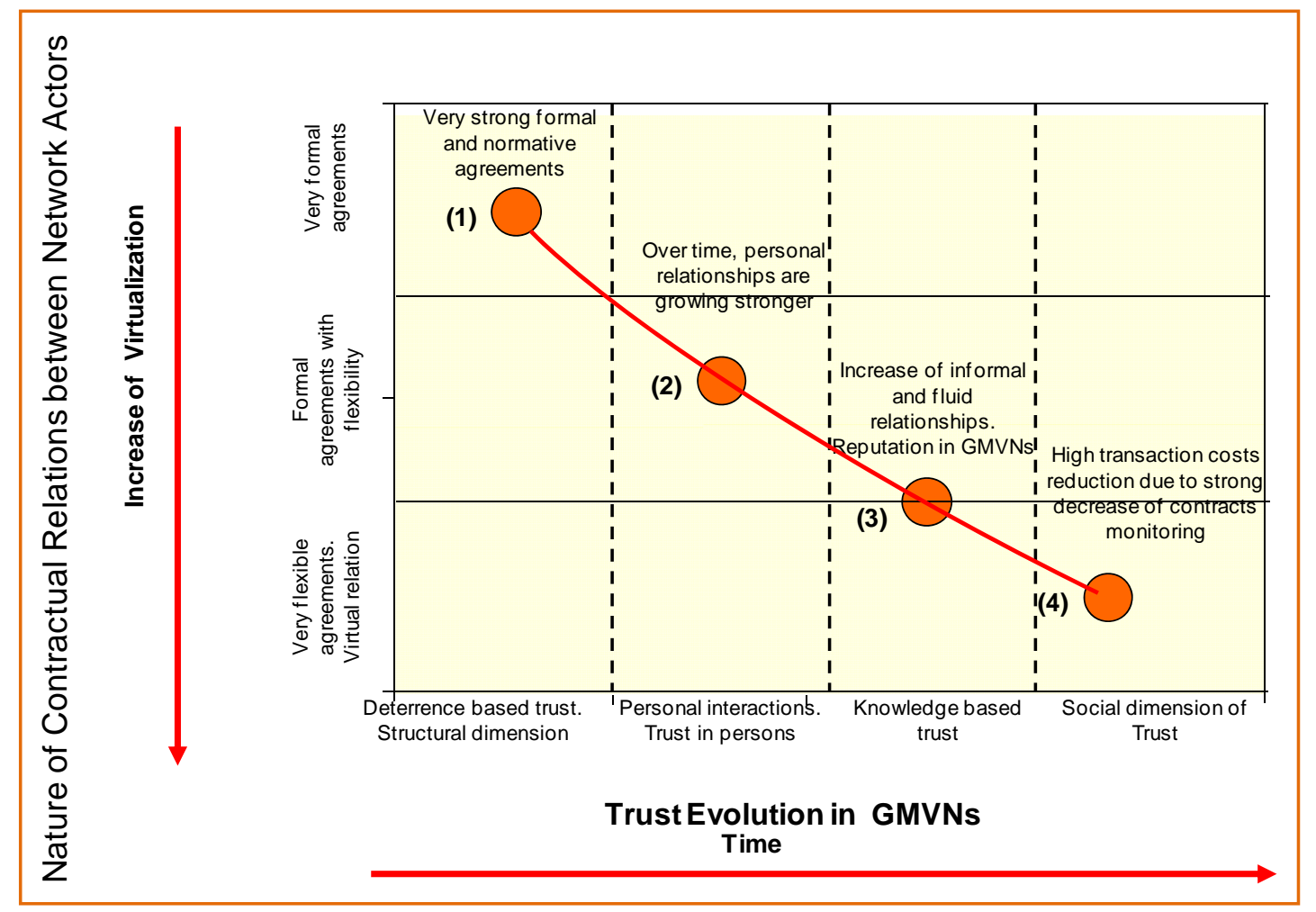

Figure 6: Relationship between Trust and "virtual" network

Proposition 2: Similarity of cultural practices between actors in GMVNs increases chances of survival and constitutes an ex ante cultural mechanism that favors the existence of homogeneous culture in the network.

Proposition 3: Trust and the degree of virtualization in a GMVN increase proportionally with time in GMVNs that are successful.

Proposition 4: An intense tacit knowledge transfer between GMVN actors increases the similarity of cultural practices, helps to homogenize culturally the network, and builds a systemic culture. 


\section{Intangible Capital}

Strategy is a fundamental building block in the design of GMVNs. In a globalized environment, the strong component of horizontal collaborations among their members may question the validity of a "Porterian" strategy based on the search for a balance of forces in the sector and on achieving a competitive advantage that is sustainable in time. In this type of network it is very common that network actors collaborate in a specific project while they compete in other projects or stages of their value chain. Dell and Compaq both compete in the hardware development and manufacturing processes, and cooperate with software companies like Netscape and Microsoft. Increasingly, OEMs collaborate with contract manufacturers due to economies of scale or specialization that they get by making similar products for several competitor OEMs. This paradox is permitted by the OEMs, because the benefits obtained through this collaboration are much greater than the inherent risks of collaborative manufacturing. This close collaboration between competitor OEMs in the same sector is very frequent in GMVNs. Thus, strategy in GMVNs must move away of classical patterns like the two-dimensional Porterian model and try to find new ways of strategy as the "coopetition" (Nalebuf \& Brandenburg, 1996).

One way to solve this paradox is by dividing the strategy of GMVNs into three perspectives: 1) Nodal strategic perspective based mainly on the traditional strategy approach without considering the benefits of external collaborations; 2) Dyadic strategic perspective based on collaborations with other companies in the network to achieve beneficial scenarios for all parties that avoid opportunistic behavior; and 3) Systemic strategic perspective based on the vision of the network as a homogeneous system where the high level of trust and how GMVN activities may fit together will permit them to compete efficiently in the market against other networks.

\section{Nodal Strategic Perspective}

A very useful tool within this context is the three-dimensional matrix (Shi et al. 2003). This matrix shown in Figure 7 is based on three independent variables that are necessary to consider when designing a manufacturing strategy in a GMVN: 1) Internationalization of the manufacturing process, 2) Supply and value chain and 3) Strategic alliances: 
Internationalization of the manufacturing process: The manufacturing process is no longer considered as one single production centre, but rather has to include expansion or dispersion plants in accordance with the company's current strategy.

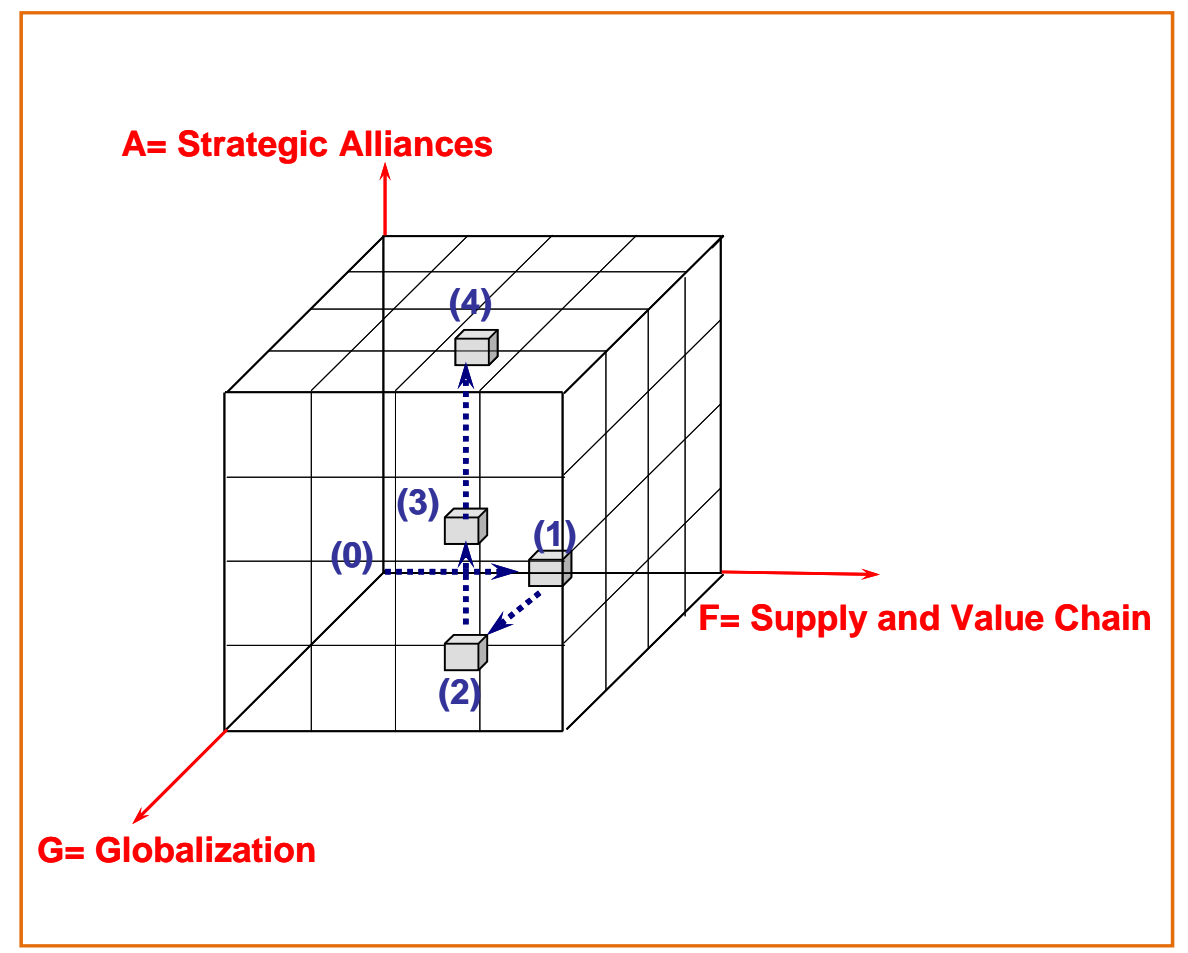

Figure 7: Strategic Positioning Of Rolls Royce Global Manufacturing Virtual Network

Supply and value chain: The various tasks involved in the manufacturing systems and carried out on the network must be defined throughout the product value chain, and both the stage of the chain during which the tasks are to be carried out and the party by whom they are to be controlled must be specified. In addition, this comprehensive view of the process will enable optimization through the selection of internal and external activities, collaborators and the types of controls established, slightly increasing process efficiency and obtaining more competitive advantages.

Strategic alliances: A very broad range of possible forms of intercompany collaboration must be assessed, ranging from specific collaborations on certain projects to long-term joint ventures or strategic alliances.

Point (0) of figure 7 refers to Rolls-Royce's beginnings in 1953, when it started up its aircraft engine manufacturing activity with a model called Dart, manufactured 


\section{Intangible Capital}

entirely at one of its plants in the United Kingdom. Point (1) shows the decentralization of the manufacturing processes begun by RR after it was privatized in 1987. Point (2) indicates the company's internationalization after privatization; however, this process did not include any significant collaboration with other companies. Point (3) shows the current situation, which presents a highly globalized company with manufacturing centres distributed all over the world (each centre specializing in one or more engine subunits), integrated supply chains that add value and a high level of participation in international projects in collaboration with other companies to form an authentic virtual network.

The aim of this network is to satisfy a number of requirements that previously limited its expansion policy, such as the possibility of incorporating technological innovations into its engines, reducing financial risks with regard to new engine projects, reducing its own manufacturing resources by subcontracting subunits to collaborating companies, with each centre specializing in one type of engine technology, and achieving economies of scale by the production process of each manufacturing centre on the network specializing in one or more engine components or subunits. In addition, by reducing its manufacturing resources, Rolls-Royce has become more flexible with regard to reacting to changes in market demand, maintaining the main competences of its organization, which, within the scope of the manufacturing process, include the design and development of the engines and the assembly and final testing stages.

Rolls-Royce's strategy on the network is to change the supply chain in accordance with the project or engine type in question. In the case of its Trent engine family, there is very little horizontal collaboration, since it has a highly consolidated position on the market and has been manufactured for many years. The manufacturing process is carried out at Rolls-Royce manufacturing centres or companies in which the company has significant holdings in the share capital. Each centre makes one or more engine components that are finally assembled and tested at its facilities in Derby in the United Kingdom. However, in the case of the new F136 engine, the supply chain is based on horizontal collaboration with manufacturers, in some cases, direct competitors (e.g., GE), to form an authentic virtual network whose external participants work together on the specific manufacture of the engine. The benefits of this type of collaboration are beyond 


\section{Intangible Capital}

question since they allow an approach to high-financial-risk projects, resulting in greater technical specialization of the components and a highly flexible production.

Point (4) in figure 5 indicates the company's trend for the coming years, where external collaboration will be used more and more to the point where the company's own resources shall be decapitalized. The future of this type of network is not based on the internal maintenance of manufacturing resources to satisfy unpredictable variations in demand. Rather it is based on relations with the various components of a virtual network that allow the company to design a specific supply chain in accordance with each engine type or client. Therefore, this type of network is not based on the possession of certain own resources that condition what can be produced, when it can be produced and how much, but rather on managing and sharing the network resources.

This three-dimensional matrix is a useful tool in the nodal perspective since permits to gain a clear and broader vision of the manufacturing strategy of a GMVN actor. Even though does not consider the effects and complexity of interacting with other network participants.

\section{Dyadic Strategic Perspective}

Under this perspective, the focus moves away from the company and is located in the collaboration itself. It is necessary to understand the motivations and needs of participants in the relationship to minimize the risk of opportunistic behavior if we want to be successful. One of the most relevant theories about this approach is what Nalebuff and Brandenburg (1996) named "coopetition". It is based on the simultaneous adoption of competitive and collaborative strategies with the market players. "Coopetition" is a relationship based on the total net value provided by all actors that participate in a business relationship including suppliers, customers, substitutes (competitors) and complementors represented in a value net. The aeroengine manufacturers Rolls Royce and GE Avio collaborate intensively in research programs with the University of Bristol or in the F136 engine manufacturing to power the F-35 Lightning II military aircraft. Nevertheless, they also compete aggressively to supply the engine that powers the new Airbus 380 with the Rolls Royce Trent 900 engine against the GP 7200 engine manufactured by a consortium where GE is present. However, under the Aristotelian logic that dominates business 


\section{Intangible Capital}

management rationality, this approach may provide a paradox difficult to be solved since a strategy of collaboration and competition with the same company leads to reconsider the rules of interaction between firms.

The theory of "coopetition" is based on the mutual benefits obtained from a potential collaboration between competitors when it is considered the entire network of value in a relationship including all stakeholders. This contribution has been very useful by giving a new perspective to the classic competitive approach but does not solve the cognitive conflict inherent in this type of collaboration. It implicitly assumes that the global economic benefit of all participants in the relationship will be enough to overcome the cognitive and emotional barriers that arise when working with competitors. However, it is necessary to design some regulatory mechanisms within the relationship that minimize this dilemma that arises when working with competitors. The absence of an approach that considers the soft factors within a collaboration is what has led to the failure of many strategic alliances between competitors. It is therefore necessary a complementary approach that takes into account the more informal or emotional aspects of collaboration.

Many scholars have addressed this problem under different approaches (e.g., Zeng \& Chen 2003; Chen \& Yao, 2003). For example, structural mechanisms seek to solve this dilemma by changing the structural parameters of the collaboration. One of the most interesting contributions in this line is by changing the structure of the pay-off matrix through a variation of the prisoner's dilemma by applying the game theory (Zeng \& Chen 2003). Other interesting contributions are by introducing structural systems of sanctions that prevent opportunistic behavior or by reducing the size of the group. However, another perspective, the motivational approach is the one that has obtained the best results. It is based on changing the perception of the network actors on the social environment. This theory shows how personal communication drastically increases cooperation so every participant can understand the nature of the dilemma and the negative consequences for the group of opportunistic behavior. Once everybody gets the picture about the different options depending on each point of view those that best promote the interest of the network will be applied. 


\section{Intangible Capital}

\section{Systemic Strategic Perspective}

A third strategic perspective refers to the strategic positioning of the whole network as a homogeneous system that competes against other manufacturing networks or individual companies. Under this strategic approach, network actors may achieve a global overview where they can fit its internal resources in a specific combination to obtain a competitive advantage difficult to imitate over time.

This complementary of activities in the context of GMVNs refers to what Doz and Hamel (1998) called "cospecialization" and implies that GMVN actors focus on a few key skills and activities that are complementary to those of other network participants. This approach involves improving the knowledge or ability on a specific field and letting other network actors to develop the other complementary activities. The more "cospecialization" is achieved the greater economies of specialization. Network participants become increasingly less similar as they become less active in technological areas transferred to other GMVN actors. The more "cospecialization", the greater dissimilarity in technological capabilities and the greater network virtualization. However, the main risk of "cospecialization" is to lose the ability to make a complete product. In case of GMVN failure, actors who have lost such a capacity must reorient its strategy even though this change does not use to be feasible within a short term. Therefore, only under a systemic approach based on trustful relationships among its network actors is plausible an intense "cospecialization" within GMVNs.

When GMVNs reach this systemic perspective, they achieve the paradigm of sustainable competitive advantage based on "cospecialización" as the way its activities fit and reinforce one another (Porter, 1996). Also they avoid the so-called "trap of the Red Queen" (Barnett \& McKendrick, 2004). This metaphor is based on the book by Lewis Carroll, "Alice through the Looking-Glass" where Alice says that although she is running as much as she can she remains in the same spot to which Red Queen replies, "it takes all the running you can do, to keep in the same place". This metaphor describes the co-evolutionary process in which companies must aggressively compete to survive. Under this perspective, strongest actors increase selection pressures, yielding more fit survivors, which in turn generate stronger competition and so on in a self-accelerating process of reciprocal causality. As suggested by the Red Queen's metaphor relative competitive position of network 
actors can be stable even though it may take high investments in $R \& D$, marketing or new product development. The systemic strategic perspective avoids this trap since each network actor specializes in some unique processes or components complementary to other network actors where activities fit and reinforce one another in a self-reinforcing process to achieve a sustainable competitive advantage against other firms or external networks.

Proposition 6: The higher degree of cospecialization in a GMVN, the higher efficiency and competitiveness will be achieved.

Last GMVN's building block is dynamics. This concept refers to network operations including areas such as information and communication technologies (ICTs) or control and monitoring tasks. Many authors have studied this field like the works of $\mathrm{Li}$ et al (2004) on manufacturing grids or Liu and Shi (2008) on collaborative manufacturing in sectors such as computer manufacturing, astronomy and bioinformatics. These works study how to coordinate the design and operation of heterogeneous manufacturing resources distributed throughout the network. Recently, several scholars (e.g., Camarinha et al., 2009; Chituc et al., 2009) have developed some conceptual models referred to the necessary ICTs to manage this type of organizations. However, there are still many aspects to develop in this area like the dilemma of how supervising these networks.

Several theories have developed the question about the need for these networks to be or not controlled. For example, the evolutionary theory of organizations (Foster, 2000) raises the dilemma between a self-organized process against other process consciously directed.

However, GMVNs have a number of characteristics that make them different from other virtual organizations. Their most relevant feature is the intense horizontal collaborations between competitors. Any effort to supervise and control these organizations will go against their original nature and will create internal tensions that could put at risk the network survival. Therefore, GMVNs should not be directed or controlled but they should be able to emerge and evolve on their own towards a dynamic equilibrium. Although in practice, it is usual that environmental factors cause substantial changes in the network morphology by producing an evolution to open structures with many weak ties and structural holes. In addition, some actors may seek centralized positions to achieve certain control or power 
over the network. Since these factors may weaken trust among network actors and strongly affect network performance, it is necessary to design some mechanisms to avoid these situations. I mplementation of ICT systems at network level can help to overcome this problem. Paradoxically, these control mechanisms will assist to prevent that network evolves towards centralized or hierarchical typologies to precisely avoid that nobody controls and manages the GMVNs. Diagram of figure 8 includes a conceptual model of ICTs in GMVNs based on multiagent technology to manage their dynamics, positions of power and to increase trust among their actors.

\section{CONCEPTUAL MODEL OF ICTS IN GMVNs}

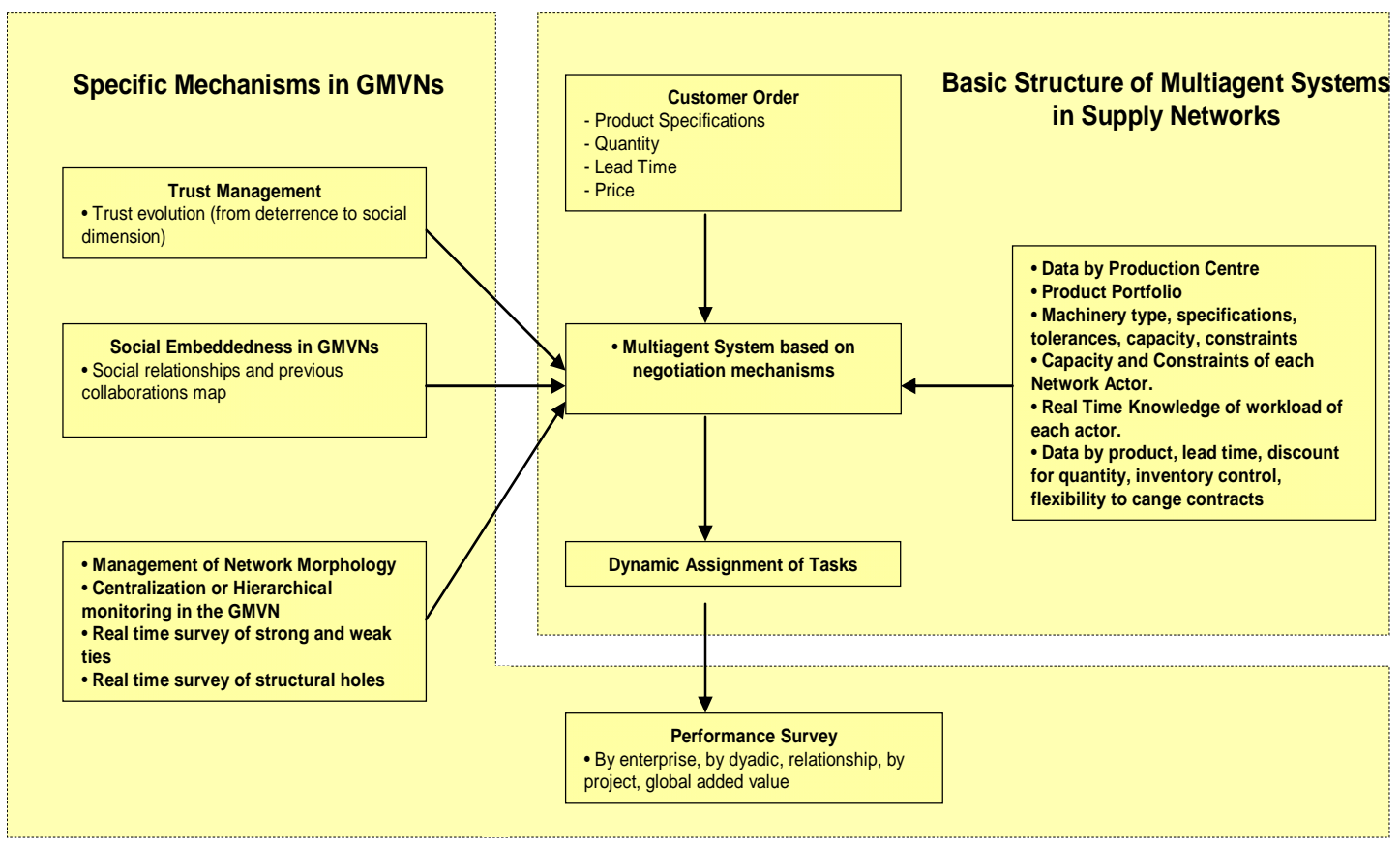

Figure 8: Conceptual Model of ICT in GMVNs

Proposition 7: GMVNs with global ICTs platforms will have less risk to move into centralized or hierarchical structures, and will reduce the threat of opportunistic behavior.

Benefits of GMVNs are unquestionable. They provide a highly efficient system characterized by low barriers to entry and exit, geographic flexibility, low cost, very fast network technology diffusion, high diversification through contract manufacturers and exceptional economies of scale and specialization. Therefore, 


\section{Intangible Capital}

this type of organization is increasingly growing in sectors such as automotive, computers, aeronautics and chemistry. However, there are a number of risks inherent in this kind of collaboration with competitors that must be avoided to ensure the survival of the network. The four proposed building blocks, structure, culture, strategy and dynamics, permit to analyze all relevant factors within these organization in a systematic manner as well as to prevent problems that can undermine their efficiency. Closed structures with many strong ties between network actors avoid opportunistic behavior but reduce innovation potential of these networks. ICTs can minimize these problems through applications that make more transparent the actors' behavior. Systemic strategy can provide a very high level of competitiveness if based on "cospecialzación" although it is important to build trust among all network actors. The importance of four cultural mechanisms described will be critical for the survival of GMVNs. If actors within these organizations are able to overcome the inherent risks of these collaborations, GMVNs will have a big growth in the coming years where it is not needed to maintain internal manufacturing resources but to manage and share the network resources.

\section{References}

AHUJ A, G. (2000). Collaboration Networks, Structural Holes, and Innovation: A longitudinal study. Administrative Science Quarterly, 45(3): 425-455.

ARRUÑADA, B.; VÁZQUEZ X. (2006). When your contract manufacturer becomes your competitor, Harvard Business Review (September).

BARNETT W.P.; MCKENDRICK, D.G. (2004). Why are some organizations more competitive than others? Evidence from a Changing Global Market. Administrative Science Quarterly, 49(4): 535-571.

BURT, R. (1992). Structural holes: The social structure of competition. Harvard University Press, Cambridge, MA.

CAMARINHA-MATOS, L. M.; AFSARMANESH, H.; GALEANO, N.; MOLINA, A. (2009). Collaborative networked organizations - Concepts and practice in manufacturing enterprises. Computers \& Industrial Engineering, 57(1): 46-60. 


\section{Intangible Capital}

CHEN, X. P.; YAO, X. (2003). Sustaining cooperation in public goods dilemmas: Effects of motivational explanations and sanctions. Paper presented the annual meeting of the Academy of Management, Seattle.

CHITUC, C.M.; TOSCAZO, C.; AZEVEDO, A. (2008). Interoperability in collaborative networks: Independent and industry-specific initiatives - The case of the footwear industry. Computers in Industry, 59: 741-757.

DOZ, Y.L.; HAMEL, G. (1998). Alliance advantage. Harvard Business Press.

ELMUTI, D.; KATHAWALA, Y. (2001). An overview of strategic alliances. Management Decision, 39: (205-217).

FINE, C. (1998). Clockspeed - Winning industry control in the age of temporary. Advantage. New York: Perseus Books.

FOSTER, J. (2000). Competitive selection, self organisation and Joseph A Schumpeter. Journal of Evolutionary Economics 10(3): 311-328.

GRANOVETTER, M. (1985). Economic action and social structure: The problem of embeddedness. The American J ournal of Sociology, 91(3): 481-510

GULATI, R. (1995). Does familiarity breed trust? The implications of repeated ties for contractual choice in alliances. Academy of Management Journal, (38)1: 85112.

GULATI, R.; GARGIULO M. (1999). Where do interorganizational networks come from?. The American Journal of Sociology, 104(5): 1439-1493.

GUPTA, A. K.; GOVINDARAJAN, V. (2000). Knowledge flows within multinational corporations. Strategic Management Journal, 21(4): 473-496.

HOFSTEDE, G.; NEUIJEN, B.; OHAYV, D.; SANDERS, G. (1990). Measuring organizational cultures: A qualitative and quantitative study across twenty cases. Administrative Science Quarterly, Vol. 35, No. 2, June, pp. 286-316.

LI, R.; YU, T.; FANG, M. (2004), The reliability management of manufacturing grid, CIMS and robot center, Shanghai University, Shanghai, China, Flexible Automation and Intelligent Manufacturing, FAIM2004, Toronto, Canada. 


\section{Intangible Capital}

LIU, Q.; SHI, Y. J. (2008). Grid manufacturing for cross-enterprise. International J ournal of Advanced Manufacturing Technology, 36:205-212.

LUO, Y. (2007). A competition perspective of MNC-host government relations. J ournal of International Management, 10(4): 431-451.

NALEBUFF, B. J.; BRANDENBURGER, A. M.; (1996). La competition, une révolution dans la manière de jouer concurrence et cooperation, Village Mondial, Paris.

POTHUKUCHI, V.; DAMANPOUR, F.; CHOI, J.; CHEN, C.C.; PARK, S.H. (2002). National and organizational culture differences and international joint venture performance. Journal of International Business Studies, 33: 243-265.

SHI, Y.; FLEET, D.; GREGORY, M. (2005). Global manufacturing virtual network and its position in manufacturing systems. The 7th Annual International Manufacturing Symposium, Institute for Manufacturing, Dept. of Engineering, University of Cambridge.

SHI, Y.; GREGORY, M. (2003). From Original Equipment Manufacturers to Total Solution Providers: an emergence of Global Manufacturing Virtual Network in electronics industry. International Journal of Service Technology and Management, 4(4-6): 331-346.

STURGEON, J. (2002). Modular production networks: A new American model of industrial organization. Industrial and Corporate Change, 11(3): 451-496.

ZENG, M.; CHEN, X.P. (2003). Achieving Cooperation in Multiparty Alliances: A Social Dilemma Approach to Partnership Management. Academy of Management Review, 28(4): 587-605.

(c) Intangible Capital, 2009(www.intangiblecapital.org)

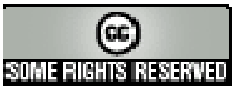

El artículo está con Reconocimiento-NoComercial 2.5 de Creative Commons. Puede copiarlo, distribuirlo y comunicarlo públicamente siempre que cite a su autor y a Intangible Capital. No lo utilice para fines comerciales. La licencia completa se puede consultar en http://creativecommons.org/licenses/by-nc/2.5/es/ 